\title{
Adverse drug reactions and the respiratory system
}

\author{
P Bhatia, J F O'Reilly, E Li-Kam-Wa
}

\section{INTRODUCTION}

Many drugs can cause respiratory toxicity. Drugs may affect the lung parenchyma, pleura, airways, mediastinum, pulmonary vasculature or the neuromusculature and hence drug induced lung problems may present in various ways. The diagnosis of drug induced lung disease is one of excluding other pathologies as imaging studies or histology may not be diagnostic. It is important to recognise the condition as cessation of treatment may lead to complete cure whereas continuation of the offending agent may lead to a fatal outcome. This article reviews the various manners in which drugs may affect the respiratory system adversely.

\section{METHODS}

We performed a search of Medline and consulted the Textbook of Respiratory Medicine ( $2^{\text {nd }}$ edition) edited by John F. Murray and Jay A. Nadel. From the obtained articles we carried out a crossover search (ie we obtained more references from bibliographies of the textbooks and papers) to write this review.

\section{Mechanisms of drug-induced lung injury $y^{1,2,3}$}

At present four mechanisms are recognised by which drugs may damage the lungs.

Oxidant injury: This type of lung injury is particularly related to the adult respiratory distress syndrome. It also plays a significant role in pulmonary fibrosis due to nitrofurantoin. Nitrofurantoin generates oxygen radicals such as hydrogen peroxide $\left(\mathrm{H}_{2} \mathrm{O}_{2}\right)$, hydroxyl radical $(\mathrm{OH})$, superoxide and singlet oxygen, which disrupt critical cell functions. These radicals are normally removed by the antioxidants (eg. Glutathione).

Nitrofurantoin and other drugs may accelerate the production of these oxygen radicals in the lung cells

Direct cytotoxic effects on alveolar capillary endothelial cells: Research with bleomycin led to postulation of this mode of lung injury. The drug is allowed to accumulate within the epidermal cells of the lungs as they contain lower levels of a specific inactivating enzyme as compared to epithelial cells in other organs. The accumulated drug passes through the nuclear membrane and results in fragmentation of the DNA and damage. ${ }^{2}$ People developing lung damage may be congenitally deficient in the inactivating enzyme.

\section{Phospholipid deposition within cells: Certain drugs} such as amiodarone cause accumulation of abnormal alveolar macrophages containing phospholipids. ${ }^{3}$ This may be due to inhibition of phospholipid catabolism and the process is reversible with discontinuation of the drug and corticosteroid therapy.

\section{Table 1: Spectrum of pulmonary toxicity that drugs may cause}

1) Alveolar haemorrhage:

2) ARDS

3) Broncholitis obliterans

4) Broncholitis obliterans

with organising pneumonia

5) Bronchospasm

6) Cough

7) Granulomatous disease

8) Immune complex

9) Interstitial fibrosis

10) Lymphocytic interstitial

11) Mediastinal lipomatosis

12) Pleural effusion

13) Pulmonary alveolar proteinosis

14) Barotrauma

15) Pulmonary eosinophilia

16) Pulmonary hypertension

17) Pulmonary venoocclusive disease

18) Inappropriate respiratory muscle contraction

19) Respiratory failure

(neuromuscular)
Penicillamine, cocaine, anticoagulants, nitrofurantoin, haloperidol, hydralazine, amphotericin $\mathrm{B}$, mitomycin, sulfonamides Amphotericin B, Acetylsalicylic acid, Bleomycin, Epinephrine, Fibrinolytics, Heroin, Hydrochlorothiazide, Methotrexate, Nitrofurantoin, Protamine, Tricyclic antidepressants, oxygen in high concentration, NSAIDs,

Oesophageal variceal sclerotherapy Penicillamine

Gold, penicillamine, sulfasalazine, bleomycin, amiodarone,methotrexate, cyclophosphamide, cocaine Beta blockers, acetylsalicylic acid, adenosine, cocaine, dipyridamole, hydrocortisone, nitrofurantoin, NSAID, protamine, propafenone, vinblastine, nebulised pentamidine, nebulised beclomethasone, vinblastine with mitomycin, ACE inhibitors, proton pump inhibitors, amiodarone, xamoterol, acipimox, tropisetron ACE inhibitors, podophyllin, zotepine, citalopram Aspiration of oily nasal drops or eye lubricants, talc (magnesium silicate), intravenous and intranasal cocaine, methotrexate

Hydralazine, procainamide, isoniazid, mediated lung disease penicillamine, hydantoins (drug induced SLE)

Busulfan, chlorambucil, cyclophosphamide, melphalan, bleomycin, methotrexate, etoposide, vinblastine, amiodarone, tocainide, nitrofurantoin, sulfasalazine, aspirin, gold, penicillamine, heroin, methadone, talc, isoniazid, hydralazine, procainamide, radiation treatment Flecainide, phenytoin, captopril disease Corticosteroids

Amiodarone, bromocriptine, chemotherapeutic agents (methotrexate), practolol, dantrolene, methysergide, nitrofurantoin, tocolytic agents (terbutaline, ritoridine \& other betamimetic drugs), oesophageal variceal sclerotherapy with sodium tetradecyl sulphate or ethanolamine oleate. pergolide Busulfan

(Pneumothorax, Pneumomediastinum and pneumopericardium) cocaine

Ampicillin, aspirin, inhaled beclomethasone, bleomycin, carbamazepine, captopril, chlorpromazine, clofibrate, cocaine, dapsone, ethambutol, fenbufen, gold, imipramine, methotrexate, metronidazole, minocycline, naproxen, nitrofurantoin, penicillin, penicillamine, pentamidine, phenytoin, propranolol, sulphonamides, sulfasalazine, tamoxifen, tetracycline, trazodone, tolazamide.

Mitomycin, aminorex, phenformin, fenfluramine, smoking cocaine, intravenous use of amphetamine from medicinal nasal inhalers

bleomycin, mitomycin, zinostatin, bischloroethyl nitrosourea, etoposide, cyclophosphamide Neuroleptics, strychnine poisoning

Calcium channel blockers, beta-blockers, diuretics, antirheumatics, aminoglycoside antibiotics, polymyxin antibiotics 
See accompanying editorial by Anthony Avery [pp 26].

\section{Dr. Praveen Bhatia Staff Physician}

\section{Dr. J.F.O'Reilly}

Consultant Physician

\section{Dr. E. Li-Kam-Wa}

Consultant Physician

Correspondence to:

Dr Praveen Bhatia Dept. of Chest Medicine Blackpool Victoria Hospital

Whinney Heys Road Blackpool FY3 8NR

\section{Naviapo@aol.com}

Date Submitted:30:04:01

Date Accepted:11:06:01

Prim Care Respir J 2001: 10(2); 39-43
Immune system mediated injury: Drug induced lupus erythematous may affect the lungs. Certain drugs incite the formation of antibodies against histones and these result in pulmonary damage. ${ }^{4}$

Damage by the above mechanisms may result in the appearance of evidence of interstitial disease on the chest radiograph. Drugs may also result in pulmonary toxicity without an abnormal chest radiograph. Table 1 lists the spectrum of pulmonary toxicity that drugs may cause.

To reach to a diagnosis, it is important to take a detailed history including purchase of over the counter medications, use of alternative medicine, drug history, any history of overdoses or past addiction. It should be ascertained whether the onset of symptoms correlates with any recent prescription. Chest X-ray and CT scan may show non-specific features and may not be diagnostic. Pulmonary function tests may reveal airflow obstruction or a restrictive disorder with altered transfer factor and hence though not diagnostic, may point to the type of disorder and can help to monitor the disease. Histology may show specific features of toxicity from certain drugs and hence may be helpful in confirming the diagnosis.

\section{Types of drug induced lung disease}

\section{Alveolar haemorrhage}

The causes of alveolar haemorrhage $e^{5}$ can be divided into those accompanied by pulmonary capillaritis and those without involvement of the pulmonary vessels. Penicillamine is usually associated without pulmonary capillaritis. Usually patients have been on at least $1 \mathrm{gm}$ daily for at least 10 months, although cases been reported after 20 years of treatment. It may be accompanied by crescentic glomerulonephritis. ${ }^{6}$

The most prominent symptoms are cough, dyspnoea, and haemoptysis. Patients may complain of chest pain. The chest radiograph demonstrates diffuse or focal alveolar infiltration. The CT scan confirms the presence of an air-space filling disorder. The lung function tests may show a raised diffusing capacity despite an abnormal chest radiograph and CT scan. Serial measurement of the diffusing capacity is a sensitive indicator in detecting active alveolar bleeding. There may be associated iron deficiency anaemia. The white blood cell, platelet count and sedimentation rate are usually raised. Bronchoalveolar lavage will reveal abundant red blood cells and haemosiderin-laden macrophages. Early treatment with haemodialysis, plasmapheresis and immunosuppression may prevent a fatal outcome. Other causes associated with alveolar haemorrhage which need to be excluded are Wegener's granulomatosis, systemic necrotizing vasculitis, Goodpastures syndrome, Behçet's syndrome, Henoch-Schönlein purpura, SLE, coagulation disorders, mitral stenosis, pulmonary veno-occlusive disease, lymphangioleiomyomatosis, idiopathic pulmonary haemosiderosis and pulmonary capillary haemangiomatosis.

Adult respiratory distress syndrome

Certain drugs can induce noncardiogenic pulmonary oedema $^{7,8}$ (increased permeability oedema). This may be due to an increase in the permeability of the alveolar capillary membrane, due to neurogenic factors or due to aspiration which is a common feature of respiratory failure of any cause.

Patients present with dyspnoea and cough. Tachypnoea is an early sign and wheeze is commonly heard. These signs are also commonly seen in increased pressure oedema, however history and physical examination may help differentiate the two conditions as patients with increased permeability oedema do not have signs of cardiac disease (raised JVP, cardiac enlargement, gallop rhythm, heart murmur, enlarged and tender liver and peripheral oedema)

Treatment may require adequate life support with ventilatory support. The causative drug should be identified and stopped. Any aggravating factor such as infection should be sought and treated. While administering oxygen, it's toxicity should be borne in mind. Adequate cardiac output and perfusion of the vital organs should be maintained.

Bronchiolitis obliterans and bronchiolitis obliterans with organising pneumonia (BOOP)

Bronchiolitis obliterans was first described in 1835. It is characterised by fibrous scarring of the small airways and intraluminal polyps in the small airways. ${ }^{9}$ Since then it has been associated with toxic fume inhalation, infection, bone marrow transplantation, lung and heart-lung transplantation, rheumatoid arthiritis, SLE and penicillamine ingestion. It is characterised by cough and dyspnoea and physical examination may reveal wheeze. The chest radiograph in bronchiolitis obliterans may be normal or may show a miliary or diffuse nodular pattern. In bronchiolitis obliterans, a flow volume loop characteristically shows a reduction in expiratory flow at low lung volumes. Penicillamine induced bronchiolitis obliterans responds poorly to corticosteroids. $\mathrm{BOOP}^{10}$ is the term used when there is extension of granulation tissue into the alveoli. BOOP induced by bleomycin is frequently nodular and may mimic metastasis. ${ }^{11}$ Bleomycin exposure with subsequent exposure to high inspired oxygen is associated with an increased incidence of lung toxicity. Hence patients who have had bleomycin in the previous 6 months should ideally not receive high flow oxygen.

\section{Bronchospasm ${ }^{12}$}

Beta-adrenergic receptors can be divided into excitatory beta-1-receptors located in the heart and inhibitory beta-2-receptors located in the bronchi. Body plethysmography can be used to demonstrate increased airway resistance in normal persons and in asymptomatic asthmatic patients on beta-blockers. Beta-blockers in the form of eye drops (Timolol) are known to have resulted in fatal cases of status asthmaticus. Timolol in eye drops is absorbed through the conjunctiva and bypasses the liver resulting in a higher concentration than if given orally. Propafenone, a membrane-stabilising antiarrhythmic agent can cause bronchospasm. Dipyridamole can cause a severe bronchospasm in less than $1 \%$ of the patients in whom it is used. It can be 
prevented by the simultaneous use of aminophylline. Vinblastine when used along with mitomycin can result in bronchospasm. Non-steroidal anti-inflammatory agents (NSAIDs) and ACE inhibitors can also cause bronchospasm.

\section{Cough}

ACE inhibitors can cause a dry and persistent cough, which is unresponsive to cough suppressants. This may occur in up to $15 \%$ of patients receiving the drugs and is twice as common in women as in men. ${ }^{13}$ The onset of cough may not be immediate. Changing to another member of the family is unhelpful. ACE inhibitors inhibit the breakdown of bradykinins and other kinins, which accumulate and cause the dry cough. It may respond to nebulised comolyn sodium. Podophyllin preparations used for treating warts have also been associated with cough.

\section{Granulomatous Disease}

Talc (magnesium silicate) is used as filler in many medications for oral use. Drug addicts may crush tablets such as methadone, propoxyphene, amphetamines and mepiridine and inject them intravenously. The talc in these preparations can result in granulomatous interstitial fibrosis, granulomatous pulmonary arterial occlusion or both. ${ }^{13,14}$ Patients present with dyspnoea, cough, symptoms of pulmonary hypertension, exercise induced syncope, right heart failure or sudden death. The chest radiograph may be normal. However a diffuse nodularity, which develops into focal nodules particularly in the upper lobe, may be seen. The lower lobes may show bullae formation and these can rupture resulting in pneumothorax. The earliest abnormality in the lung function test is a low diffusing capacity. Pulmonary tissue may show granulomatous change with multinucleated giant cells and fibrosis. Talc can be detected by the presence of strongly birefringent crystals using polarised light. Bronchoalveolar lavage may reveal increased lymphocytosis and both intracellular and free talc. Half of these patients also develop talc retinopathy characterised by the presence of talc emboli within blood vessels near the macula. ${ }^{15}$ A trial of corticosteroid should be given although results of therapy vary from none to significant improvement. Use of oily nasal or ophthalmic drops is also associated with the development of granulomatous reaction if aspirated. CT scanning may help in diagnosing infiltrates secondary to aspiration of oily ophthalmic preparations. $^{16}$

Methotrexate given in low doses once or twice a week is also associated with a granulomatous interstitial pneumonitis in about $5 \%$ of patients. There is synergy with chronic nitrofurantoin use and patients on methotrexate should therefore not receive nitrofurantoin. ${ }^{17}$

\section{Immune complex mediated lung disease}

A small percentage of patients on the drugs mentioned in table 1 develop the pleuropulmonary syndrome associated with immune complex mediated lung disease. Patients develop antinuclear antibodies (primarily against histones). ${ }^{18}$ Symptoms develop insidiously and the patient usually has been on the offending drug for many months or even years.
Patients complain of myalgia, fever polyarthralgia, pleurisy and cutaneous lesions. Renal involvement is rare. A chest radiograph may show basal infiltrates, pleural effusions, atelectasis, or cardiomegaly secondary to pericardial effusion. Symptoms usually resolve on stopping the offending drug and corticosteroids may be used if resolution is not rapid or if the drug cannot be stopped for clinical reasons. The abnormal serum antibodies disappear in a few months. ${ }^{19}$

\section{Interstitial Fibrosis}

Interstitial fibrosis secondary to certain drugs presents as dyspnoea and cough. The chest radiograph may be normal or show diffuse interstitial changes.

Methotrexate pneumonitis may show mediastinal, hilar lymphadenopathy or pleural effusion in $10-15 \%$ of patients. ${ }^{20}$ Clubbing has not been recorded with drug induced interstitial lung disease. Auscultation of the lungs discloses crackles but these may also be seen in infections, heart failure and cryptogenic fibrosing alveolitis. The carbon monoxide diffusing capacity may decrease before a fall in lung volumes. Gallium uptake may be increased before the chest radiograph becomes abnormal.

Amiodarone induced interstitial fibrosis occurs in up to $6 \%$ of the patients taking over $400 \mathrm{mg}$ of the drug daily for two or more months. This may be fatal in 10$20 \%$ of patients. ${ }^{21} \mathrm{CT}$ scan of the chest shows an increase in pulmonary density in areas of accumulation. Increased density of the liver is also noted on the CT scan. It can also cause mass-like lesions, which may cavitate. ${ }^{22}$

\section{Lymphocytic interstitial disease $\mathrm{e}^{23,24}$}

This is characterised by lymphocytic infiltration in the lung interstitium. It is also associated with autoimmune disorders (Sjögren's syndrome, primary biliary cirrhosis, pernicious anaemia, chronic active hepatitis, SLE, myasthenia gravis), AIDS, bone marrow transplantation, hypogammaglobulinemia \& tuberculosis. Besides the features of the underlying condition, patients present with cough, progressive dyspnoea, pleuritic chest pain, and fever. Patients may be clubbed or cyanosed. The chest radiograph shows nonspecific reticulonodular infiltrates. Lung biopsy is essential for diagnosis and shows extensive lymphocytic infiltration involving the alveolar septa, peribronchiolar and perivascular interstitium. Corticosteroids may bring about complete resolution.

\section{Mediastinal Lipomatosis ${ }^{2.5}$}

This is an unusual complication of corticosteroid administration. It may also be seen in obese patients. There is deposition of fat in the mediastinum and on chest radiograph, which appears as a widened mediastinum mimicking lymphadenopathy or other neoplasia. Patients usually have a Cushingoid appearance and $\mathrm{CT}$ of the chest demonstrates a fatty consistency of the mediastinal mass. The mediastinal fat does not compromise vital structures and hence the steroids may be continued. 


\section{Pleural Effusion}

Certain drugs are known to cause pleural effusion. It is usually unilateral and small in size. In some patients it may be due to drug induced SLE and an antinuclear antibody assay should be performed. Pleural effusion secondary to bromocriptine therapy may be accompanied by lymphocytosis. ${ }^{26}$ It usually starts two months to four years after initiation of bromocriptine and is reversible after discontinuation of the drug. Oesophageal variceal sclerotherapy with sodium tetradecyl sulphate, ethanolamine oleate or sodium morrhuate can result in an abnormal chest radiograph showing pleural effusion in $85 \%$ of the patients. Development of a pleural effusion depends upon the volume of sclerosing agent injected and has been reported in up to $50 \%$ of patients. Most patients remain asymptomatic. However fever and chest discomfort have been reported. Other pulmonary complications of sclerotherapy are ARDS, atelectasis, mediastinal widening and pulmonary infiltrates.

\section{Pulmonary Alveolar Proteinosis}

This rare disorder characterised by the deposition of amorphous, insoluble, proteinaceous material in the alveoli and bronchioles has been described in patients suffering from chronic myelogenous leukaemia ${ }^{27}$ and receiving busulphan. ${ }^{28}$ Epithelial hyperplasia caused by busulphan is probably responsible for the excessive surfactant production. The primary disturbance is impairment in oxygen transfer to the blood perfusing the alveoli and patients present with hypoxemia. Unlike spontaneous primary pulmonary alveolar proteinosis, it may not respond to whole-lung lavage.

\section{Barotrauma}

Barotrauma has been reported with the use of 'crack' cocaine. ${ }^{29}$ This is due to shearing forces on alveolar structures allowing free air to dissect up the fascial planes. Several factors may contribute to its occurrence. 'Crack' cocaine addicts may inhale from a small pipe aggressively and often perform a Valsalva manoeuver prior to exhaling against pursed lips. This is alleged to enhance the euphoria but can result in barotrauma. Pneumothorax may also be produced by direct puncture of the mediastinum and chest structures during intravenous injections. Treatment is generally supportive and chest drainage may be needed in symptomatic patients.

\section{Pulmonary eosinophilia ${ }^{30}$}

Several drugs can cause pulmonary eosinophilia; the most common are nitrofurantoin, non-steroidal antiinflammatory drugs, antibiotics, and anti-neoplastic drugs. Patients may present with cough, dyspnoea and fever. Patients with drug-induced eosinophilic lung disease can vary in presentation from a mild syndrome to a fulminant condition. Chest radiograph may show pleural effusion or lung infiltrates. Bronchoalveolar lavage shows an increase in the percentage of eosinophils present. Although many patients will improve on discontinuing the offending drug, corticosteroids can hasten recovery in severe cases.

\section{Pulmonary hypertension ${ }^{31}$}

Pulmonary hypertension has been associated with certain medicinal compounds. ${ }^{29}$ Intravenous use of amphetamines obtained from medicinal nasal inhalers has been known to cause pulmonary artery foreign body granulomas. Pulmonary artery medial hypertrophy is known to occur with cocaine smoking. Cocaine can also cause pulmonary vascular smooth muscle vasoconstriction.

\section{Pulmonary venoocclusive disease $e^{32}$}

Pulmonary veno-occlusive disease results from obstruction of small veins and venules and has been described as a complication of chemotherapy for malignant disease.$^{33}$ Patients present with dyspnea, haemoptysis, syncope and signs of cor pulmonale. Pulmonary artery catheterisation reveals an elevated pulmonary capillary wedge pressure in addition to pulmonary hypertension. Lung function tests show a decrease in carbon monoxide diffusing capacity with preservation of lung volumes. Definitive diagnosis is by lung biopsy, which demonstrates fibrous intimal obliteration of venules. Other causes are idiopathic or autoimmune with features of Raynaud's phenomenon, arthritis, and raised serum autoantibodies.

\section{Inappropraite respiratory muscle contraction ${ }^{34}$} Strychnine is a common diluent of many street drugs. It blocks the inhibitory neurones in the spinal cord such that motor neurone discharge can be precipitated by trivial sensory stimuli. The symptoms may begin within minutes of ingestion and patients are usually alert. Treatment is supportive. Diazepam or phenobarbitone can control milder spasms. In severe toxicity it may be necessary to paralyse the patient using pancuronium or d-tubocurarine or general anaesthesia. Excretion of strychinine occurs within 72 hours and cumulative toxicity does not occur.

\section{Respiratory failure (neuromuscular)}

Calcium channel blockers, beta-blockers, antirheumatics and diuretics can impair respiratory muscle function. ${ }^{35}$ This is usually subclinical and does not result in respiratory failure. However, respiratory failure in patients with existing impaired respiratory muscle function may occur. Aminoglycoside and polymyxin antibiotics may result in ventilatory failure.$^{36}$ This occurs by interference with neuromuscular transmission and the risk is high in patients with renal failure and myasthenia gravis. Spontaneous recovery can occur within a few hours or days of stopping the antibiotic. Anticholinesterase therapy can reverse the effects of aminoglycoside neuromuscular blockade but not polymyxin B induced neuromuscular blockade.

Organophophates, by inhibiting acetylcholinesterase lead to an accumulation of acetylcholine at the neuromuscular junction and other cholinergic synaptic sites, resulting in muscle paralysis. ${ }^{37}$ This is treated by atropine to reverse the muscarinic effects, and pralidoxime to reverse the neuromuscular junction paralysis. 
Opiods depress respiration (rate and depth) by reducing the sensitivity of the respiratory centre to a rise in blood $\mathrm{CO} 2$ tension. Patients may develop carbon dioxide narcosis.

\section{CONCLUSION}

A range of drugs may affect different areas of the respiratory system. Routine radiology, lung function and other investigations may be non-specific. For these reasons, a high degree of clinical suspicion and a detailed history with particular attention to the timing of drug ingestion and onset of symptoms are essential for diagnosis and appropriate management. Lung biopsy may help in diagnosis but may be impossible to perform if the patient is too unwell.

\section{REFERENCES}

1. Rosenow EC. Drug-induced Pulmonary Disease. Textbook of Respiratory Medicine, Murray \& Nadel. Second edition. W.B Saunders Company 1994;2118-20.

2. Adamson IY. Drug-induced pulmonary fibrosis. Environ. Health Perspect 1984;55:25-36.

3. Martin WJ, II, Rosenow E.C, III. Amiodarone pulmonary toxicity. Recognition and pathogenesis (part 2). Chest 1998 93:1242-8.

4. Schoen RT, Trentham DE. Drug-induced lupus: An adjuvant disease. Am. J. Med 1981;71:5-8.

5. Schwarz MI, Cherniack RM, King Jr. TE. Diffuse Alveolar Haemorrhage and other Rare infiltrative Disorders. Textbook of Respiratory Medicine. Murray \& Nadel. Second edition. W.B. Saunders Company 1994:1889-912.

6. Louie S, Gamble CN, Cross CE. Penicillamine associated pulmonary haemorrhage, J Rheumatol 1986;13:963-66.

7. Reed CR, Glauser FL. Drug-induced noncardiogenic pulmonary oedema. Chest 1991;100:1120-4

8. Flick MR. Pulmonary Oedema and Acute Lung Injury. Textbook of Respiratory Medicine. Murray \& Nadel. Second Edition. W.B. Saunders Company 1994:1737-62.

9. Lazarus SC. Disorders of the Intrathoracic Airways. Textbook of Respiratory Medicine. Murray \& Nadel. Second Edition. W. B. Saunders Company 1994;1473-76.

10. Epler GR, Colby TV, McLoud TC, Carrington CB, Gaensler EA. Bronchiolitis obliterans organising pneumonia. $N$ Engl J Med 1985;312:152-8

11. Bellamy EA, Husband JE, et al. Bleomycin related lung damage: CT evidence. Radiology 1985;156:155-8.

12. Rosenow EC. III. Drug-induced Pulmonary Disease. Murray \& Nadel. Second Edition. W. B. Saunders Company 1994;2139-40.

13. Rosenow EC, Myers JL, Swensen SJ, Pisani RJ. Drug-induced Pulmonary Disease An Update. Chest 1992;102:239-50.

14. Waller BF, Brownlee WJ, Roberts WC. Self-induced pulmonary granulomatosis. A consequence of intravenous injection of drugs intended for oral use. Chest 1980:78; 90-4.

15. Rosenow EC. III.: Drug-induced Pulmonary Disease. Murray \&
Nadel. Second Edition. W.B. Saunders Company 1994;2128-29.

16. Prakash UB, Rosenow EC. III: Pulmonary complications from ophthalmic preparations. Mayo Clin Proc 1990;65:521-29.

17. McKendry RJR, Cyr M. Toxicity of methotrexate compared with azathioprine in the treatment of rheumatoid arthiritis: a case-control study of 131 patients. Arch Intern Med 1989;149:685-9.

18. Rosenow EC. III: Drug-induced Pulmonary Disease. Murray \& Nadel. Second Edition. W.B. Saunders Company, 2120, 1994.

19. Cush JJ, Goldings EA. South Western Internal Medicine Conference. Drug induced lupus: Clinical spectrum and pathogenesis. Am J Med Science 1985;290:36-44.

20. Rosenow EC. III: Drug-induced Pulmonary Disease. Murray \& Nadel. Second Edition. W.B. Saunders Company1994; 2123-24.

21. Martin WJ, Rosenow EC. III.: Amiodarone Pulmonary Toxicity: recognition and pathogenesis (part I). Chest 1988;93:1067-75.

22. Piccione W Jr, Penfield Faber L, Rosenberg MS. Amiodaroneinduced Pulmonary mass. Ann Thorac Surg 1989;47:918-19.

23. Chamberlain DW, Hyland RH, Ross DJ. Diphenylhydantoininduced Lymphocytic Interstitial Pneumonia. Chest 1986;90: 458-60.

24. Kidney JC, Domhnall J, O'Halloran, Professor Fitzgerald M.X: Captopril and lymphocytic alveolitis. BMJ 1989;299:981 (letter).

25. Nguyen KQ, Hoeffel C, Le LH, Phan HT. Mediastinal lipomatosis. South-Med-J 1991;12:1169-72.

26. McElvaney NG, Wilcox PG, Churg A, Fleetham JA Pleuropulmonary Disease during Bromocriptine Treatment of Parkinson's Disease. Arch. Intern Med 1988;148:2231-36.

27. Aymard JP, Gyger M, Lavalee R, Legresley JL, Desy M. A case of pulmonary alveolar proteinosis complicating chronic myelogenous leukaemia. Cancer 1980;46:1763-6.

28. Watanabe K, Sueishi K, Tanaka K et al. Pulmonary alveolar proteinosis and disseminated atypical mycobacteriosis in a patient with busulfan lung. Acta Pathol Jpn 1990;40:63-6.

29. Albertson EA, Walby WF, Derlet RW. Stimulant induced pulmonary toxicity. Chest 1995;108:1140-9.

30. Allen JN, Davis WB. Eosinophilic lung diseases. Am J Respir Crit Care Med 1994;150:1423-38.

31. Kumar K, Holden WE. Drug-induced pulmonary vascular disease mechanisms and clinical patterns. West J Med 1986:145;343-49.

32. Schwarz MI, Cherniack RM, King TE Jr. Diffuse Alveolar Haemorrhage and other rare infiltrative disorders. Murray \& Nadel. Second Edition. W.B.Saunders Company. 1994;1900-2.

33. Lambard CM, Churg A. Winokur S. Pulmonary Veno-Occlusive Disease following Therapy for Malignant Neoplasms. Chest 1987;92:871-86.

34. Aldrich TK, Rochester DF. The lungs and neuromuscular Diseases. Murray \& Nadel. Second Edition. W.B. Saunders Company. 1994;2516.

35. Aldrich TK, Prezant DJ. Adverse effects of drugs on the respiratory muscles. Clin Chest Med 1990;11:177-89.

36. Lindesmith LA, Baines RD Jr, Bigelow DB et al. Reversible respiratory paralysis associated with polymixin therapy. Ann Intern Med 1968;68:318-27.

37. Taylor P. Anticholinesterase agents. Gilman AG, Gilmer LS, Rall TW et al. The Pharmacological Basis of Therapeutics. New York, MacMillan, 1985:110-29. 